\title{
Cannibalism as a selective force on offspring size in fish
}

\author{
Olsson, Karin; Andersen, Ken Haste
}

Published in:

Oikos

Link to article, DOI:

10.1111/oik.04131

Publication date:

2018

Document Version

Peer reviewed version

Link back to DTU Orbit

Citation (APA):

Olsson, K., \& Andersen, K. H. (2018). Cannibalism as a selective force on offspring size in fish. Oikos, 127(9), 1264-1271. https://doi.org/10.1111/oik.04131

\section{General rights}

Copyright and moral rights for the publications made accessible in the public portal are retained by the authors and/or other copyright owners and it is a condition of accessing publications that users recognise and abide by the legal requirements associated with these rights.

- Users may download and print one copy of any publication from the public portal for the purpose of private study or research.

- You may not further distribute the material or use it for any profit-making activity or commercial gain

- You may freely distribute the URL identifying the publication in the public portal

If you believe that this document breaches copyright please contact us providing details, and we will remove access to the work immediately and investigate your claim. 
Manuscript type: Research paper

2

Title: Cannibalism as a selective force on offspring size in fish

\section{Authors}

6 Karin H. Olsson ${ }^{1,2, *}$, email: olsson.karin.h@gmail.com

ORCID iD, 0000-0002-1695-0989

8 Ken Haste Andersen ${ }^{1}$, email: kha@aqua.dtu.dk

\section{Affiliations}

1: Center for Ocean Life, National Institute for Aquatic Resources, Technical University of

12 Denmark, Bygning 202, DK 2800 Lyngby, Denmark

2: Cefas Laboratory, Lowestoft, Suffolk NR33 0HT, United Kingdom (present address)

14

* Corresponding author

16

Keywords: cannibalism, offspring size, life history

18 


\section{Abstract}

Cannibalism may cause considerable mortality on juvenile fish and it has been hypothesised that it

may exercise selection on offspring size in that larger offspring may enjoy a size refuge. For this to be evolutionarily advantageous the survival of individual offspring must compensate for the

24 reduced fecundity implied by larger offspring size. We develop a model which combines standard assumptions of size-dependent mortality with adult cannibalism to investigate the potential for

26 cannibalism to act as selective force on offspring size. We find that for this potential to be realised, the mortality due to cannibalism must exceed a threshold value that is a decreasing function of non-

28 cannibalistic predation intensity, cannibalized size range width and the average cannibalized size. If cannibalism exceeds this threshold, the model predicts evolution of offspring size towards refuges

30 above or below cannibalized size range depending on initial offspring size. Cannibalistic mortality cannot be so great that the population is non-viable, however, the range of parameter values

32 describing cannibalistic intensity allowed within these boundaries is wide. On this basis, we suggest that cannibalism is a potential mechanism for offspring size selection. 


\section{Introduction}

Offspring size is an important component of reproduction. Larger offspring typically enjoy greater survival (Baker 2008) but at the cost of reduced parental fecundity (Elgar 1990, Berrigan 1991). In aquatic organisms, predators are often gape-size limited and mortality therefore decreases with size (Peterson and Wroblewski 1984, Houde 1997). While the prediction that mortality declines with specific population. If, for example, the abundance or voraciousness of a segment of predators is predators. Such variation in size-dependent mortality may influence selection on optimal offspring 

1992) and Baltic Sea cod Gadus morhua (Neuenfeldt and Köster 2000) was consistent with adult prey size preference. The impact of cannibalism can be high, potentially removing up to $95 \%$ of an age class in some aquatic organisms (Fox 1975). In Baltic Sea cod, cannibalism may remove 44\% of a cohort in the first two years (Neuenfeldt and Köster 2000), although the level of cannibalism varies over time and with spawning stock biomass (Jensen and Sparholt 1992). Cannibalism has also been linked to low food concentration (Folkvord 1991, Nilsson et al. 2011), though food availability and adult population size are likely to covary (Smith and Reay 1991). Due to the sizespecific nature of cannibalism, in combination with the potential impact on juvenile survival, it has been put forward as a source of selection on offspring size (Weeks and Gaggiotti 1993, Nilsson et al. 2011).

To investigate the circumstances under which cannibalism can exercise selection on offspring size we build a lifetime fitness model which specifically includes cannibalistic mortality. We base our model on the assumption of a non-growing population and identify the evolutionary endpoint strategy. First, we assume that all density-dependence occurs early in life and treat cannibalistic mortality as a free parameter, recognising that the level of cannibalism may vary with many external factors. Second, we assume that cannibalism acts as the sole density-regulating mechanism, and use this criterion to determine the level of cannibalistic mortality.

78

\section{Lifetime fitness model}

80 We build an evolutionary life history model assuming a non-growing population at equilibrium to explain the selective pressure cannibalism may exercise on offspring size. We assume a two-stage

82 life cycle consisting of a juvenile phase during which all available energy is used for growth and an adult phase where the energy is directed to reproduction, such that growth is determinate. All

84 individuals are assumed to be affected by density-independent predation mortality, but only adults 
are cannibalistic. Juveniles, therefore, also experience mortality due to adult cannibalism. We ignore the energetic benefit adults may obtain from the predation and further assume that the number of juveniles is sufficiently large so that an adult is unlikely to reduce its fitness by consuming its own offspring. To parameterise the model, we make the following assumptions of growth, mortality and reproduction (all model parameters are listed in Table 1):

1. Growth rate scales metabolically with size (body weight) $w$

$$
g(w)=A w^{n}
$$

where $A$ is a growth constant and $n$ is the metabolic exponent (von Bertalanffy 1957, Day and Taylor 1997, West et al. 2001) which we set to $n=3 / 4$.

2. Predation mortality rate is assumed to be a power law scaling with size as

$$
\mu_{p}(w)=a A w^{\mathrm{n}-1}
$$

where $a$ is a non-dimensional parameter giving the strength of predation. Kokkalis et al. (2017) estimated values for $a$ for a range of fish stocks and found these to be lognormally distributed with mean 0.22 and standard deviation 0.7 in the log domain; the model will be explored for values $0.1<a<0.8$. Predation mortality is also assumed to be proportional to the growth constant $A$ as higher foraging rates imply higher mortality (empirically, e.g. Sih 1982, theoretically, Werner and Anholt 1993). In fish, empirical data suggest that mortality typically scales with size as $w^{-0.25}$ (Peterson and Wroblewski 1984, McGurk 1986). The model becomes mathematically simpler if juvenile and adult mortality have the same scaling, though relaxing this assumption does not qualitatively change the outcome, as shown in Appendix A.

3. Cannibalism is assumed to increase mortality for sizes around the adult preferred predator-prey size ratio $\beta$. We model this preferred prey size using an Ursin size selection function 


$$
\theta\left(w / w_{m}\right)=\exp \left[\frac{\log ^{2}\left(\beta w / w_{m}\right)}{2 \sigma^{2}}\right]
$$

where $w_{m}$ is the adult size and $\sigma$ defines the width of the selection function. For fish, typical parameter values are $\sigma=1$ and $\beta=100$ (Ursin 1973). We assume that cannibalistic mortality rate is proportional to the size selection $\mu_{c}(w)=c_{c} \theta\left(w / w_{m}\right)$. It turns out that the notation later is much simplified if we define a dimensionless constant $b=c_{c} w_{m}^{1-n} / A$. With this definition, the cannibalistic mortality rate becomes

$$
\mu_{c}(w)=b A w_{m}^{n-1} \theta\left(w / w_{m}\right)
$$

where $b$ is a non-dimensional parameter describing the strength of the cannibalistic mortality. The value of $b$ depends on the number of adults and juveniles, adult prey preference and the availability of alternative food sources. We do not know the value of $b$, but explore the consequences using two approaches: fitness optimisation, where density-dependence occurs early in life such that $b$ is independent of other life history parameters, and adaptive dynamics, where $b$ is determined from the population structure.

124 4. Growth is assumed to be determinate: adults direct all available energy to reproduction. The available energy at the adult stage thus scales with size as growth does at the juvenile stage, i.e. $A w_{m}^{n}$. Therefore, consistent with empirical studies (e.g. Charnov et al. 2001), the rate of reproduction scales with size at maturity as $R_{m}=\varepsilon A w_{m}^{n}$ where $\varepsilon$ is the reproductive efficiency which accounts for energy expenditure not directly directed towards offspring production.

130 With these four assumptions, we can calculate the expected lifetime reproductive value $R_{0}$ as the probability of survival to maturation $s_{w_{0} \rightarrow w_{m}}$ multiplied with adult reproductive output $f_{m}: R_{0}=$

$132 s_{w_{0} \rightarrow w_{m}} f_{m}$. The adult reproductive output is obtained by integrating the rate of reproduction discounted by adult survival from age at maturity, $t_{m}$, to $\infty$, dividing by offspring size $w_{0}$ and multiplying by a factor of $\frac{1}{2}$ to account for an even sex ratio: 


$$
f_{m}=\frac{1}{2 w_{0}} \int_{t_{m}}^{\infty} \varepsilon A w_{m}^{n} \exp \left[-a A w_{m}^{n-1}\left(t-t_{m}\right)\right] \mathrm{d} t=\frac{\varepsilon w_{m}}{2 a w_{0}}
$$

Adult reproductive output is therefore proportional to adult body size $w_{m}$, which is consistent with empirical studies (Tsoukali et al. 2016).

Survival is calculated by integrating the ratio between mortality $\mu(w)$ and growth $g(w)$ rates from

140 size at birth to size at maturity, $s_{w_{0} \rightarrow w_{m}}=\exp \left[-\int_{w_{0}}^{w_{m}} \mu(w) / g(w) \mathrm{d} w\right]$ (Thygesen et al. 2005,

Kiflawi 2006). The total mortality is the sum of the predation mortality $\mu_{p}$ and the cannibalistic

142 mortality $\mu_{c}$ and total survival is the product of the respective survival probabilities, $s_{w_{0} \rightarrow w_{m}}=\mathrm{s}_{p} s_{c}$. Inserting growth (Eq. 1) with predation mortality (Eq. 2) gives survival due to predation $s_{p}$ as

$$
s_{p}=\left(\frac{w_{0}}{w_{m}}\right)^{a}
$$

and with cannibalistic mortality (Eq. 3) gives survival due to cannibalism $s_{c}$ as

$$
s_{c}=\exp \left[-b c \mathrm{H}\left(\mathrm{w}_{0}, \mathrm{w}_{\mathrm{m}}\right)\right]
$$

with

$$
\begin{aligned}
& \mathrm{c}=\beta^{n-1} \sigma \sqrt{\frac{\pi}{2}} \exp \left[\frac{\sigma^{2}(n-1)^{2}}{2}\right] \text {, and } \\
& \mathrm{H}\left(\mathrm{w}_{0}, \mathrm{w}_{\mathrm{m}}\right)=\operatorname{erf}\left[\frac{\log (\beta)+(n-1) \sigma^{2}}{\sigma \sqrt{2}}\right]-\operatorname{erf}\left[\frac{\log \left(\beta\left(w_{0} / w_{m}\right)\right)+(n-1) \sigma^{2}}{\sigma \sqrt{2}}\right]
\end{aligned}
$$

150 where erf is the error function. Multiplying survivals and adult reproductive output (Eq. 4) gives lifetime reproductive value $R_{0}$

$$
R_{0}=\left(\frac{w_{0}}{w_{m}}\right)^{a} \exp \left[-b c \mathrm{H}\left(w_{0}, \mathrm{w}_{m}\right)\right] \varepsilon \frac{w_{m}}{2 a w_{0}}
$$

The growth parameter $A$ disappears from the survival function and the survival depends only on the

154 non-dimensional mortality parameters $a$ and $b$ and the size-selectivity parameters $\beta$ and $\sigma$.

Furthermore, since Eq. 7 depends on the ratio between offspring size $w_{0}$ and adult size $w_{m}$, rather and adult size $z=w_{0} / w_{m}$, which we will call the relative offspring size. Simple physiology

\begin{tabular}{l} 
Formatted: Danish \\
Formatted: Danish \\
Formatted: Danish \\
Formatted: Danish \\
Formatted: Danish \\
Formatted: Danish \\
Formatted: Danish \\
Formatted: Danish \\
Formatted: Danish \\
Formatted: Danish \\
Formatted: Danish \\
Formatted: Danish \\
Formatted: Danish \\
\hline
\end{tabular}


requires $0<z<1$. Analysing the model using $z$ provides a means of generalising offspring size strategies without referring to any specific size. Eq. 7 can thus be rewritten as

$$
R_{0}=z^{a-1} \exp [-b c \mathrm{H}(z)] \frac{\varepsilon}{2 a}
$$

If $\beta$ and $\sigma$ are constants, $c$ is also constant and the fitness of a particular offspring size strategy $z$ only depends on the non-dimensional parameters reproductive efficiency $\varepsilon$, predation parameter $a$ and cannibalism parameter $b$.

\section{Optimal strategy}

166 The optimal strategy is that which leads to the highest fitness, here measured as the lifetime reproductive value $R_{0}$. Evaluating the optimal strategy in a non-growing population requires determining how density-dependence operates. If density-dependence occurs as a multiplicative factor early in life with no further effect on growth and survival, the optimal strategy can be

170 identified by locating the offspring size strategy which yields the highest fitness $R_{0}$ (Mylius and Diekmann 1995) for a given value of cannibalism parameter $b$. However, if cannibalism constitutes

172 the main density-regulating mechanism, the level of cannibalism will itself be determined as the amount of density-dependence needed to achieve a population at steady state, i.e. $R_{0}=1$. In that

174 case, we need to determine the evolutionarily stable strategy (ESS) as the strategy that cannot be invaded by other strategies (Geritz et al. 1998). Below we investigate the model under both scenarios.

\section{Early density-dependence}

As relative offspring size $z$ increases, individual survival increases, but parental fecundity decreases.

180 Cannibalism introduces a phase of elevated mortality in the size range defined by the prey size preference parameters $\beta$ and $\sigma$ (Fig. 1a). The effect of offspring size on survival is then described

182 by an increasingly $S$-shaped curve, where larger offspring size offers a refuge from the cannibalistic 
mortality phase (Fig. 1b). The optimal offspring size ratio $z^{*}$ is found by taking the first derivative of the fitness function (Eq. 8) and setting it to $0, d R_{0} / d z=0$ :

$$
\frac{\mathrm{dR}}{\mathrm{dz}}=\frac{\varepsilon \mathrm{s}_{p} s_{c}}{2 \mathrm{z}^{2}}\left(1+\frac{\mathrm{bc}}{\sigma \mathrm{a}} \overline{\frac{2}{\pi}} \exp \left[-\left(\frac{\log (\beta \mathrm{z})+(n-1) \sigma^{2}}{\sigma \sqrt{2}}\right)^{2}\right]-\frac{1}{a}\right)
$$

In the absence of cannibalism $(b=0)$, the term in the parenthesis is independent of $z$ and Eq. 9 is negative for $z$ in the allowed range $0<z<1$. Therefore, $R_{0}$ is a strictly decreasing function of $z$ and maximum $R_{0}$ is given by the smallest possible value of $z$ (Fig. 1c). This means that in the absence of cannibalism, the optimal strategy is to make as small eggs as possible. If cannibalism is strong enough, there may also be a local optimum for a larger- $z$ strategy, in addition to the small-z strategy identified by Eq. 9 if $b=0$. Whether the local, larger- $z$ optimum will occur can be determined by analysing the expression inside the brackets. The local optimum for the large- $z$ strategy is given by

$$
z^{*}=\frac{1}{\beta} \exp \left[\sigma \sqrt{\log \left(2 b^{2} c^{2} /\left(\pi \sigma^{2}(1-a)^{2}\right)\right)}+(1-n) \sigma^{2}\right]
$$

which is only defined if

$$
b \geq b_{\min }=\sqrt{\pi \sigma^{2}(1-a)^{2} /\left(2 c^{2}\right)}
$$

From Eq. 10, it follows that the local maximum $z^{*}$ decreases with increasing prey size preference $\beta$ and increases with the strength of cannibalism $b$. Consequently, there are two optimal offspring size

200 strategies: a small one and a large one, as shown in Fig. 2. For the two strategies to emerge, $b \underline{\text { must }}$ exceed a threshold value $b_{\min }-b \geq b_{\min }$, which decreases as the predation parameter $a$ increases

202 (Eq. 11, $b_{\min }$ dashed line in Fig. 2; the range of $a$ is assumed to be $0.1<a<0.8$ ).

\section{Density-dependent cannibalism}

Next, we will assume that all density-dependence is caused by cannibalism and try to locate evolutionary stable strategies (ESS) by determining when a resident strategy $z$ cannot be displaced 
by an alternative invader strategy $\tilde{z}$ (Maynard Smith and Price 1973). In a non-growing population,

the resident strategy $z$ must have fitness $R_{0}(z)=1$. The level of cannibalism required to maintain the population at equilibrium is found by rearranging Eq. 8:

$$
b_{e q}(Z)=\frac{1}{c H(z)} \log \left(\varepsilon \frac{s_{p}}{2 a z}\right)
$$

212 The evolutionary stability of the resident strategy depends on whether there exists an alternative, invader strategy $\tilde{z}$, which is rare enough not to affect $b_{e q}$ but has higher fitness than the resident $z$. This can be analysed by constructing a so-called pairwise invasibility plot (van Tienderen and de Jong 1986, Geritz et al. 1998). This plot charts the relative success of an invader strategy (along the $\mathrm{y}$-axis) against a resident strategy (along the $\mathrm{x}$-axis); by definition, the resident's $R_{0}=1$, so if the invader's $R_{0}>1$, it can invade (grey areas), while if the invader's $R_{0}<1$, it canot (white areas).resident $R_{y}=1$, so if invader $R_{y}>1$ the invader can invade (grey areas), while if invader $R_{\theta}<1$ it cannet (white areas). Note that this procedure is slightly different than the usual which

220 would calculate the invasion fitness as the population growth rate, with the criterion for invasion being that the invasion fitness $>0$ (e.g. Geritz et al. 1998). Nevertheless, as we are only interested in whether the invader can invade or not, and not the speed of invasion, the two criteria are identical. If the invader strategy is more successful than the resident, the trait value in the population will shift towards that of the invader. For small differences in size, the direction of selection can be determined by the sign of the first derivative of the fitness function of the invader $\tilde{z}$, evaluated for a particular resident strategy $z$ and therefore $b_{e q}(z)$ (Geritz et al. 1998). If the derivative is positive offspring size will increase and vice versa, as indicated with black arrows in Fig 3. When the derivative is 0 , the strategies have equal fitness, illustrated by the intersection points along the diagonal in Fig. 3. If the second derivative at the intersection point is negative, the point represents a fitness maximum for the invader. As both smaller and larger invaders have lower fitness the strategy is evolutionarily stable (ESS at $z=z_{e q}$, filled circle in Fig. 3). Conversely, if the second 
derivative is positive, both smaller and larger invader have higher fitness and the point is referred to as an evolutionary repeller (open circle in Fig. 3).

The pairwise invasibility plot in Fig. 3 indicates the possibility of two evolutionarily stable strategies, depending on the resident strategy $z$. If the resident strategy $z$ is within the size range of the adult prey size preference (indicated by the dotted line showing the Ursin selection function) and greater than the evolutionary repeller (indicated by the open circle), the gain in offspring survival increases rapidly with size, leading to selection for larger offspring as a size refuge from cannibalism. The ESS at $z=z_{e q}$ represents the evolutionary endpoint of this selection and the final trade-off between fecundity and individual offspring survival. Conversely, for resident $z$ smaller than the evolutionary repeller, larger offspring size will reduce fecundity without a sufficient gain in survival, and selection will favour ever smaller $z$.

The relative offspring size at ESS and the evolutionary repeller depends on the predation parameter $a$ and the reproductive efficiency $\varepsilon$ (Eq. 12, Fig. 4). Cannibalism will only contribute to selection for larger offspring if the level of cannibalism at equilibrium $b_{e q}$ is greater than $b_{\min }$ (as described above; Eq. 11 and dashed line in Fig. 2). Higher predation (large values of a) leads to lower density-dependent cannibalism ( $b_{e q}$ decreases with increasing $a$ ), so that eventually, at high $a$, the large offspring size ESS disappears along with the evolutionary repeller. Increasing the reproductive efficiency increases the number of surviving juveniles, which leads to higher cannibalism (consistent with the observation that cannibalism increases with larger stock size (Jensen and Sparholt 1992)). Increasing reproductive efficiency can thus compensate for increased mortality and make the ESS appear at higher mortalities.

\section{Discussion}


A key life history trade-off is that between offspring size and offspring numbers, typically representing the balance of offspring survival against parental fecundity. This constitutes the principal assumption in much theoretical work (Smith and Fretwell 1974, Kiflawi 2006) and is supported by empirical findings (Elgar1990, Blackburn 1991). Considering only this trade-off, in conjunction with metabolic scaling of growth and mortality, leads to an optimal offspring size that is infinitely small, with a correspondingly infinitely high fecundity (Thygesen et al. 2005, Kiflawi 2006). On the other hand, if mortality scales differently for juveniles and adults, optimal offspring size can be demonstrated to have a defined optimum (Kiflawi 2006, Jørgensen et al. 2011). Our model builds on this trade-off with the addition that cannibalism can also act as a driver of offspring size. The analysis of the model demonstrates that cannibalism can select for large offspring size, provided that the mortality imposed by cannibalism is strong enough to dominate other sources of mortality. It is also worth noting that in Appendix, one of the outcomes of the model is a single optimum formed from the combined effects of predation mortality and cannibalism, which suggests that cannibalism may contribute to selection on offspring size, either alone or in conjunction with other sources of mortality. Nevertheless, selection for larger offspring size may be prevented if the higher fecundity associated with producing smaller offspring compensates for the additional mortality. The conclusion from the model is therefore that two evolutionarily stable strategies may exist: small offspring size and large offspring size, where only the large offspring size strategy is driven by cannibalism.

In the case of marine animals in general and fish in particular, there is evidence for two distinct offspring size strategies: either very small compared to the adult size or roughly proportional to the adult size (Neuheimer et al. 2015, Olsson et al. 2016). The large offspring strategy, identified by

Neuheimer et al. (2015) as 'proportional', approximately matches the prey size preference 
determined by $\beta$ and is thus only slightly lower than the large- $z$ ESS that emerges from our model makes cannibalism a possible contributor to this offspring size strategy.

284 The model is kept simple to focus on size-dependent mortality and cannibalism by adults as the driving force of offspring size evolution. These simplifications ignore potentially important effects of demography, population mixing, positive effects of cannibalism and flexibility of trait evolution. For instance, compared to population demographics, evolution is typically a slow process, and for the purposes of modelling an adaptive response to evolutionary pressure we assume a population in equilibrium. Nevertheless, an underlying assumption when evaluating selection is that the magnitude of the selection factor remains reasonably constant between breeding seasons, but if cannibalism destabilises population demography (e.g. Cushing 1991, Claessen et al. 2000) or if external factors such as the availability of alternative food sources or adult mortality are highly variable, this may not be the case. Furthermore, to exercise selection on offspring size, the magnitude of cannibalism must be above a certain minimum level relative to the background mortality. Even then, selection for larger offspring would depend on the initial state of the resident population. Theoretical models have suggested that cannibalism is more likely to evolve if there is a spatial overlap between adults and juveniles (Smith and Reay 1991) and the population is wellmixed (Lion and van Baalen 2009). This suggests that the likelihood of cannibalism resulting in directional selection on offspring size may depend on habitat characteristics. For instance, Fox (1975) suggested that freshwater habitats, which are often more temporal in nature as well as and spatially unstructured, may be more likely to offer the required spatial overlap, which would help explain the prevalence of cannibalistic freshwater species. In the data compiled by Olsson et al. (2016), the teleost species that produced relatively large offspring tended to be small and inhabit brackish- and shallow-water habitats, especially in the breeding season, which would appear to be consistent with the observation made by Fox (19911975). 
Furthermore, in populations where cannibalism is substantial, juveniles may be expected to employ

counter-measures to mitigate the threat. For instance, juvenile perch can utilise refuges to reduce cannibalistic mortality (Lundvall et al. 1999) and fry survival in experimental guppy populations improves when shelters are available (Nilsson et al. 2011). If effective, for instance if the refuges offer juveniles shelter while denying adults access, larger size may no longer confer a sufficiently substantial advantage to compensate for lost fecundity and selection for larger offspring size would weaken. The model also assumes that only adults which have attained a specific size engage in cannibalism, but both inter- and intracohort cannibalism has been recorded in many fish (Smith and Reay 1991). If cannibalism is a feature over a greater range of sizes, the $\sigma$ of the model would be expected to increase, causing the 'dome' of the mortality curve in Fig. 1a to widen and become less distinct relative to other sources of mortality, with a less prominent selection on size.

On the other hand, the model ignores the positive effects cannibalism may have, both on the adults and surviving juveniles. For instance, if alternative food sources are scarce or inaccessible, cannibalism may provide adults with a ready supply of nutrients (van den Bosch et al. 1988), which would facilitate and maintain the prevalence of cannibalism in a population (Klug et al. 2006). Furthermore, surviving juveniles would benefit from less competition for food and shelter. In this model cannibalism has been assumed to be non-filial and therefore not reducing the survival of related offspring, but filial cannibalism is prevalent in fish species with parental care (Manica 2002). This may exert selective pressure on offspring if parents can selectively remove low-quality offspring (Klug et al. 2006). However, most fish are broadcast spawners, and are unlikely to be able to distinguish their own offspring once the eggs have hatched. Ultimately, the more benefits cannibalism confers upon the cannibals, the more likely the strategy will be and the more potential the selection pressures outlined in the model will have to translate into selection on offspring size. 


\section{References}

336 Amundsen, P.-A. 1994. Piscivory and cannibalism in Arctic charr. - J. Fish Biol. 45: 181-189.

Baker, J. D. 2008. Variation in the relationship between offspring size and survival provides insight into causes of mortality in Hawaiian monk seals. - Endanger. Species Res. 5: 55-64.

Berrigan, D. 1991. The allometry of egg size and number in insects. - Oikos 60: 313-321.

Blackburn, T.M. 1991. An interspecific relationship between egg size and clutch size in birds. - Auk. 108: 973-977.

342 Brown, J. H. et al. 2004. Toward a metabolic theory of ecology. - Ecology 85: 1771-1789.

Charnov, E. L. et al. 2001. Reproductive constraints and the evolution of life histories with indeterminate growth. - Proc. Natl. Acad. Sci. U. S. A. 98: 9460-9464.

Christensen, B. 1996. Predator foraging capabilities and prey antipredator behaviours: Pre- versus postcapture constraints on size-dependent predator-prey interactions. - Oikos 76: 368-380.

Claessen, D. et al. 2000. Dwarfs and giants: Cannibalism and competition in size-structured populations. - Am. Nat. 155: 219-237.

Cushing, J. M. 1991. A simple model of cannibalism. - Math. Biosci. 107: 47-71.

350 Day, T. and Taylor, P. D. 1997. Von Bertalanffy's growth equation should not be used to model age and size at maturity. - Am. Nat. 149: 381-393.

352 Elgar, M. A. 1990. Evolutionary compromise between a few large and many small eggs: comparative evidence in teleost fish. - Oikos 59: 283-287. 
Folkvord, A. 1991. Growth, survival and cannibalism of cod juveniles (Gadus morhua): effects of feed type, starvation and fish size. - Aquaculture 97: 41-59.

356 Fox, L. R. 1975. Cannibalism in natural populations. - Annu. Rev. Ecol. Syst. 6: 87-106.

Geritz, S. A. H. et al. 1998. Evolutionarily singular strategies and the adaptive growth and branching of the evolutionary tree. - Evol. Ecol. 12: 35-57.

Gislason, H. et al. 2010. Size, growth, temperature and the natural mortality of marine fish. - Fish Fish. 11: 149-158.

Holst, J. C. 1992. Cannibalism as a factor regulating year-class strength in the Norwegian springspawning herring stock. - ICES C. 1992/H14 Pelagic Fish Comm.: 1-16.

Houde, E. D. 1997. Patterns and trends in larval-stage growth and mortality of teleost fish. - J. Fish Biol. 51: 52-83.

Jensen, H. and Sparholt, H. 1992. Estimation of predation mortality of cod in the Central Baltic using MSVPA. - ICES C.M. 1992/J23 Balt. Fish Comm.: 1-41.

Jørgensen, C et al. 2011. A model for optimal offspring size in fish, including live-bearing and parental effects. - Am. Nat. 177: E119-E135.

Kiflawi, M. 2006. On optimal propagule size and developmental time. - Oikos 113: 168-173. Klug, H. et al. 2006. Parents benefit from eating offspring: Density-dependent egg survivorship compensates for filial cannibalism. - Evolution (N. Y). 60: 2087-2095.

372 Kokkalis, A. et al. 2017. Estimating uncertainty of data limited stock assessments. - ICES J. Mar. Sci. 74: 69-77.

374 Lion, S. and van Baalen, M. 2009. The evolution of juvenile-adult interactions in populations structured in age and space. - Theor. Popul. Biol. 76: 132-145. 
Lundvall, D. et al. 1999. Size-dependent predation in piscivores: interactions between predator foraging and prey avoidance abilities. - Can. J. Fish. Aquat. Sci. 56: 1285-1292.

Manica, A. 2002. Filial cannibalism in teleost fish. - Biol. Rev. Camb. Philos. Soc. 77: 261-277.

Maynard Smith, J. and Price, G. R. 1973. The logic of animal conflict. - Nature 246: 15-18.

McGurk, M. D. 1986. Natural mortality of marine pelagic fish eggs and larvae: role of spatial patchiness. - Mar. Ecol. Prog. Ser. 34: 227-242.

Mylius, S. D. and Diekmann, O. 1995. On evolutionarily stable life histories, optimization and the need to be specific about density dependence. - Oikos 74: 218-224.

384 Neuenfeldt, S. and Köster, F. W. 2000. Trophodynamic control of recruitment success in Baltic cod: the influence of cannibalism. - ICES J. Mar. Sci. 57: 300-309.

386 Neuheimer, A. B. et al. 2015. Adult and offspring size in the ocean over 17 orders of magnitude follows two life history strategies. - Ecology 96: 3303-3311.

Nilsson, K. A. et al. 2011. Guppy populations differ in cannibalistic degree and adaptation to structural environments. - Oecologia 167: 391-400.

390 Olsson, K. H. et al. 2016. Differences in density-dependence drive dual offspring size strategies in fish. - J. Theor. Biol. 407: 118-127.

392 Persson, L. 1987. The effects of resource availability and distribution on size class interactions in perch, Perca fluviatilis. - Oikos 48: 148-160.

394 Peterson, I. and Wroblewski, J. S. 1984. Mortality rate of fishes in the pelagic ecosystem. - Can. J. Fish. Aquat. Sci. 41: 1-4.

396 Polis, G. A. 1981. The evolution and dynamics of intraspecific predation. - Annu. Rev. Ecol. Syst. 12: $225-251$. 
Sih, A. 1982. Foraging strategies and the avoidance of predation by an aquatic insect, Notonecta hoffmanni. - Ecology. 63: 786-796.

400 Smith, C. C. and Fretwell, S. D. 1974. The optimal balance between size and number of offspring. Am. Nat. 108: 499-506.

402 Smith, C. and Reay, P. 1991. Cannibalism in teleost fish. - Rev. Fish Biol. Fish. 1: 41-64.

Sparholt, H. 1994. Fish species interactions in the Baltic Sea. - Dana 10: 131-162.

Thygesen, U. H. et al. 2005. How optimal life history changes with the community size-spectrum. -

Proc. R. Soc. B Biol. Sci. 272: 1323-1331.

Tsoukali, S. et al. 2016. Adult lifetime reproductive value in fish depends on size and fecundity type. - Can. J. Fish. Aquat. Sci. 73: 1405-1412.

Ursin, E. 1973. On the prey size preferences of cod and dab. - Meddr Danm. Fisk- og Havunders. 7: $85-98$.

410 van den Bosch, F. et al. 1988. Cannibalism as a life boat mechanism. - J. Math. Biol. 26: 619-633.

van Tienderen, P. H. and De Jong. G. 1986. Sex ratio under the haystack model: Polymorphism may occur. - J. Theor. Biol. 122: 69-81.

von Bertalanffy, L. 1957. Quantitative laws in metabolism and growth. - Q. Rev. Biol. 32: 217-231.

414 Weeks, S. C. and Gaggiotti, O. E. 1993. Patterns of offspring size at birth in clonal and sexual strains of Poeciliopsis (Poeciliidae). - Copeia 1993: 1003-1009.

416 Werner, E. E. and Anholt, B. R. 1993. Ecological consequences of the trade-off between growth and mortality rates mediated by foraging activity. - Am. Nat. 142: 242-272.

418 West, G. B. et al. 2001. A general model for ontogenetic growth. - Nature 413: 628-631. 
Table 1. Parameters used in the model.

\begin{tabular}{|c|c|c|c|}
\hline Parameter & Unit & Values used in model & Description \\
\hline$w_{0}$ & g & Variable & offspring weight \\
\hline$w_{m}$ & g & Variable & adult weight \\
\hline$z$ & - & $0<z<1$ (variable) & relative offspring size \\
\hline$n$ & - & $3 / 4$ (fixed, West et al. 2001) & metabolic scaling exponent \\
\hline$A$ & $\mathrm{~g}^{1-\mathrm{n}} /$ time & $5 \mathrm{~g}^{1 / 4} \mathrm{yr}^{-1}$ (Fig. 1, otherwise non-specified) & growth constant \\
\hline$\varepsilon$ & - & $\varepsilon=0.1, \varepsilon=0.2, \varepsilon=0.4$ (fixed) & reproductive efficiency \\
\hline$f_{m}$ & - & variable (non-specified) & adult lifetime fecundity \\
\hline$a$ & - & $0.1 \leq a \leq 0.8$ (variable, Kokkalis et al. 2017) & predation parameter \\
\hline$b$ & - & $0 \leq b$ (variable $)$ & cannibalism parameter \\
\hline$\beta$ & - & 100 (fixed, Ursin 1973) & preferred predator/prey size ratio \\
\hline$\sigma$ & - & 1 (fixed, Ursin 1973) & narrowness of the selection function \\
\hline$C$ & - & $\approx 0.41$ (fixed) & constant defined by $\beta$ and $\sigma$ (Eq. 6 ) \\
\hline
\end{tabular}

424 Figure legends
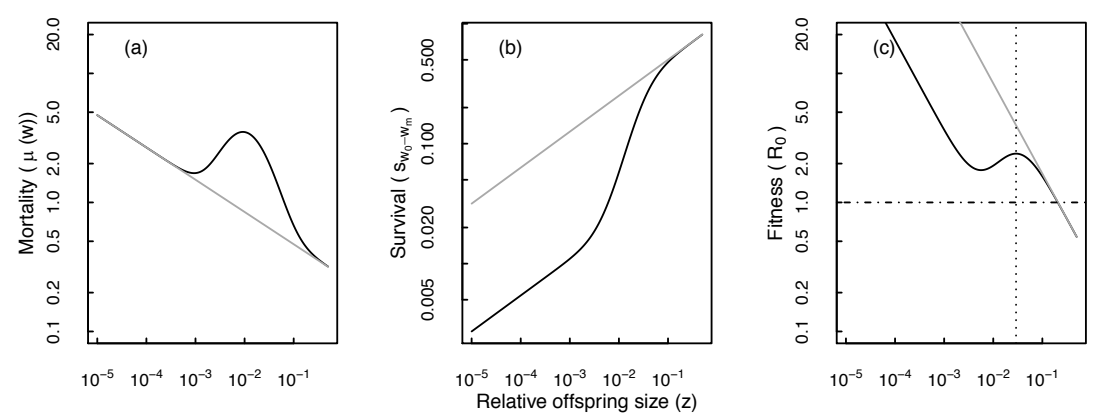
The effect of cannibalism according to the model on a) mortality at size $\left(\mu(w)=\mu_{p}(w)+\mu_{c}(w)\right)$,

b) survival to maturity $\left(s_{w_{0} \rightarrow w_{m}}\right)$, and c) lifetime fitness $\left(R_{0}\right)$. Dashed vertical line shows the local large- $z$ optimum. Parameter values are $a=0.3, A=5 \mathrm{~g}^{1 / 4} \mathrm{yr}^{-1}$, and $w_{m}=1000 \mathrm{~g}$. Grey lines correspond to $b=0$ and black lines to $b=3$.

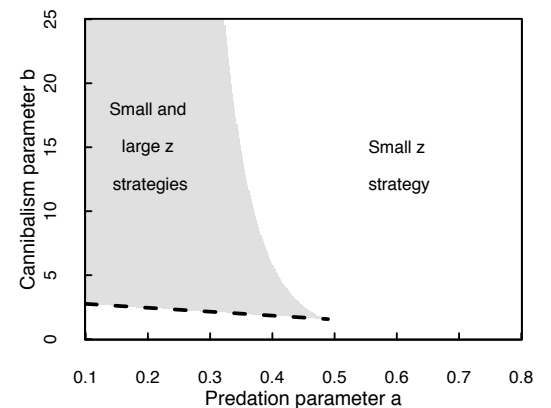

Fig 2

434 The combined influence of predation parameter $a$ and cannibalism parameter $b$ on optimal offspring size for reproductive efficiency $\varepsilon=0.2$. Combinations of $a$ and $b$ resulting in selection for small offspring only is shown in white while combinations resulting in both a small offspring and a local, large offspring, optimum strategy are shown in grey. Dashed line shows $b=b_{\min }$.

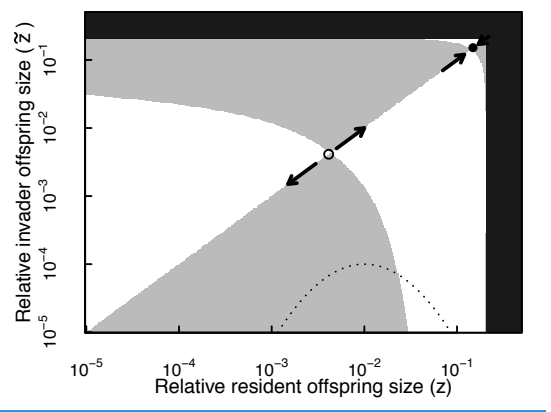


$440 \quad$ Fig 3

Pairwise invasibility plot showing the evolutionary outcome for density-dependent cannibalism.

442 Predation parameter $a=0.3$ and reproductive efficiency $\varepsilon=0.2$. Grey areas show $R_{0}(\tilde{z})>\mathrm{R}_{0}(z)$, white areas $R_{0}(\tilde{z})<\mathrm{R}_{0}(z)$ and black areas $R_{0}(z)<1$. ESS points are identified by filled circles while open circles show the evolutionary repeller and arrows the direction of selection. The dotted line shows the corresponding Ursin selection function.

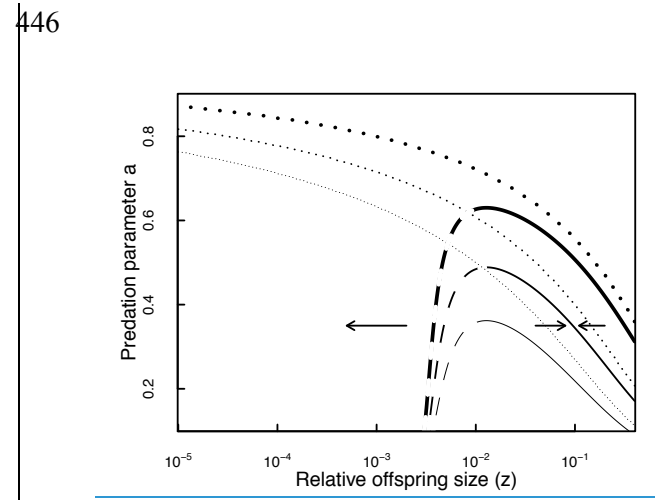

$448 \quad$ Fig 4

Effect of predation parameter $a$ on equilibrium strategies for three values of reproductive efficiency $450 \varepsilon$ (thin lines $\varepsilon=0.1$, medium lines $\varepsilon=0.2$, thick lines $\varepsilon=0.4$ ). ESS and evolutionary repeller are indicated by solid and dashed lines, respectively. Arrows show the direction of selection for $\varepsilon=0.2$.

452 Dotted lines show the corresponding limits for $R_{0}(z)=1$. 
458 Juvenile mortality may scale differently from adult mortality, which would mean that juvenile survival decreases faster with decreasing size than has been assumed above. Formulating the model

460 for a different scaling, juvenile predation mortality can be expressed as

$$
\mu_{p}=a A w_{m}^{n-1}\left(\frac{w}{w_{m}}\right)^{n-1+d}
$$

462 which approaches the expected size scaling for adults $n-1$ as size $w \rightarrow w_{m}$. The additional nondimensional parameter $d$ describes the relative difference in scaling between growth and mortality in juveniles. For $d=0$, the model is the same as above. If $d \neq 0$, the survival function is given by

$$
s_{p}=\exp \left[\frac{a}{d}\left(\left(w_{0} / w_{m}\right)^{d}-1\right)\right]
$$

466 Using the relative offspring size $z=w_{0} / w_{m}$, fitness can be expressed as

$$
R_{0}=\exp \left[\frac{a}{d}\left(z^{d}-1\right)\right] \exp [-b c \mathrm{H}(z)] \frac{\varepsilon}{2 a z}
$$

and fitness of a given offspring size strategy $z$ depends on reproductive efficiency $\varepsilon$, predation parameter $a$, the additional mortality scaling parameter $d$ and the cannibalism parameter $b$. The derivative of Eq. A. 2 is

$$
\frac{\mathrm{d} R_{0}}{\mathrm{~d} z}=\frac{\varepsilon s_{p} s_{c}}{2 z^{2}}\left(z^{d}+\frac{b c}{\sigma a} \sqrt{\frac{2}{\pi}} \exp \left[-\left(\frac{\log (\beta z)+(n-1) \sigma^{2}}{\sigma \sqrt{2}}\right)^{2}\right]-\frac{1}{a}\right)
$$

Optimal offspring size is given by setting $d R_{0} / d z=0$. In the absence of cannibalism $(b=0)$ this becomes $z^{*}=a^{-1 / d}$, i.e. not indefinitely small, contrary the model result above. For positive values of $b$, Eq. A.3 cannot be solved analytically, but there are three possible scenarios: 1) no effect of cannibalism, 2) two local optima representing a small- $z$ and a large- $z$ strategy with either the small$z$ or the large- $z$ strategy as the global optimum and 3) a single optimum, determined by the combined values of $a, d$ and $b$ (Fig. A1a). For low values of $a$ (or $d$ close to 0 ), optimal offspring strategy $z^{*}=a^{-1 / d}$ is much smaller than the size range targeted by cannibalism and two local 
fitness optima will occur, provided that $b$ is high enough, similar to when $d=0$. Higher values of $b$

480 have the potential to eliminate the viability of the small-size optimum at $z^{*}=a^{-1 / d}$ as $R_{0}<1$, reducing the viable fitness curve to a single optimum. For higher values of $a, z^{*}$ is within the target range of cannibalism, and the effect of higher cannibalism $b$ will be to shift the optimum upwards.

As before, finding stable ESS requires calculating the first and second derivative of Eq. A.2 with respect to $\tilde{z}$. The corresponding pairwise invasibility plot (Fig. A1b) shows three equilibrium strategies $z_{e q}$, two of which are ESS but separated by an evolutionary repeller. The lower-z ESS corresponds to that of $z=a^{-1 / d}$ (dotted line in Fig. A1b). Thus, different scaling of mortality will still allow cannibalism to select for either smaller or larger offspring size, depending on the initial conditions, where the offspring strategy is to seek refuge either above or below the targeted size range.

\section{Figure legend}

Fig. A1

494 Direction of selection on offspring size when juvenile mortality scales differently $(d=-0.15)$. In a) the combined influence of predation parameter $a$ and cannibalism parameter $b$ : white indicates selection for small offspring only, light grey for two fitness optima with smaller $z$ having higher fitness, medium grey for two fitness optima with larger $z$ having higher fitness and dark grey for a single optimum for large $z$ only. In b) the pairwise invasibility plot shows the evolutionary outcome of density-dependent cannibalism for $a=0.3$ and $\varepsilon=0.2$ : grey areas show $R_{0}(\tilde{z})>\mathrm{R}_{0}(z)$ and white areas $R_{0}(\tilde{z})<\mathrm{R}_{0}(z)$, filled circles show ESS, open circles the evolutionary repeller, and arrows direction of selection. Dotted line is drawn for the smaller optimal offspring size ratio $z^{*}=$

$502 a^{-1 / d} \approx 0.00033$. In both, black areas indicate $R_{0}(z)<1$. 